\title{
Effect of Landslide on Energy Efficiency Chain Based Routing Protocol for Wireless Sensor Network
}

\author{
Vu Van Khoa, Shigeru Takayama \\ Graduate School of Science and Engineering, Ritsumeikan University, Shiga, Japan \\ Email: gr0184sr@ed.ritsumei.ac.jp, s-tkym@st.ritsumei.ac.jp
}

How to cite this paper: Khoa, V.V. and Takayama, S. (2020) Effect of Landslide on Energy Efficiency Chain Based Routing Protocol for Wireless Sensor Network. Wireless Sensor Network, 12, 13-36. https://doi.org/10.4236/wsn.2020.122002

Received: February 5, 2020

Accepted: February 26, 2020

Published: February 29, 2020

Copyright (๑) 2020 by author(s) and Scientific Research Publishing Inc. This work is licensed under the Creative Commons Attribution International License (CC BY 4.0).

http://creativecommons.org/licenses/by/4.0/

\section{(c) (i) Open Access}

\begin{abstract}
Wireless sensor network has been used as a landslide monitoring tool for more than one decade. The robustness of the network is important as the systems need to survive in harsh conditions. In this paper, we consider the living time of the sensor network under the influences of the small-scale landslide. We investigate the performance of famous energy-efficient routing protocol PEGASIS in both landslide case and non-landslide case. Genetic Algorithm is also applied to enhance the effectiveness of PEGASIS. The simulation results in this paper showed that the Genetic Algorithm helps to delay the first node death if it is used at the beginning of data transmission while being used every round helps to prolong last node death slightly. The impact of the Genetic Algorithm on energy usage and route length is also examined. Under the effect of landslide, with only $70 \%$ of energy are spent, the simulated protocols reduced around 30\% equivalent route length while managed to keep the living time up the network up to $90.76 \%$, comparing to cases with no landslide.
\end{abstract}

\section{Keywords}

Wireless Sensor Network, Landslide, Routing Protocol

\section{Introduction}

The Wireless sensor network (WSN) is recognized as a good candidate for monitoring the environment as well as the natural phenomenon. For environment monitoring applications, WSN is useful in observing the familiar matters, including living conditions of animals and plants, indoor and outdoor air quality, to larger objectives, for example, forest fire or climate change [1]. With appro- 
priate sensors, a WSN can help to manage some kinds of disasters, for instance: flood, landslide, snow avalanche.

For landslide disaster monitoring, researchers have used different sensors and various methods to detect the parameters which directly or indirectly cause the landslides to happen [2] [3] [4] [5] Many studies focus on developing the hardware of sensor nodes [2] [5] and the deployment of the network [2] [3] [5] [6]. The essential of a sensor node for landslide monitoring is emphasized with the robust structure to withstand certain damage, and the ability to quickly analyze and transmit data during emergencies. The nodes share the same characteristic with the ones of other applications: low energy and limited possibility of energy harvesting.

The studies in landslide monitoring applications usually observe local case studies and utilize local geographical information [5] [6]. Geographic Information System (GIS) is a useful tool to access local geographical information at almost any location on Earth [7]. With the assistance of GIS, some potential landslides can be discovered, and local authorities can verify the risk probability by examining the area in detail. As mentioned in [8], the landslide zoning process may contain sources of errors, including errors in information of landslide trigger events, preparatory factors, and description of the area. To improve the accuracy of the information, the sensor node network can be employed to measure parameters at specific locations. Under large-scale landslides, most of the nodes in the network may be destroyed, rendering the system ineffective. In these cases, the camera systems, GIS, or other approaches are more useful. WSN is more suitable for smaller-scale landslides where there is a need of continuous gathering information, even a portion of the network is affected and incapable of functioning normally.

A landslide occurrence may not only damage one node or a part of WSN but also sweep them out of original locations. However, as most of the proposed systems set the node at a determined location, WSN for landslide monitoring is considered as a static network. The routing protocol applied for this kind of network is mainly based on the hierarchical arrangement of the nodes. Because energy efficiency is one of the important aspects in WSN, there are classical routing protocols considered it as the main target. LEACH is the representative of classic cluster-based and PEGASIS is the one of chain-based routing protocol [9] [10]. In [11], LEACH, PEGASIS, and other protocols have been reviewed in terms of network living time and PEGASIS shows an excellent result.

Despite both routing protocol and landslide get attentions of WSN researchers, the combination of these two topics does not have many works. In [12], some cluster-based protocols are examined in considered with landslide situation; however, the result does not present the impact of the incidents. The chain-based routing protocol in general and PEGASIS in particular, have not been examined with respect to landslide. While most of the landslide-related studies have not covered the chain-based routing protocol, we would like to investigate how PEGASIS works under the risk of landslide occurrence. This re- 
quires a representation of landslide in an appropriate simulation to PEGASIS, which have not been studied by related works.

In this paper, we separate a large-scale landslide into a series of small-scale landslides to assess the effects over time. The impact of landslides may shorten the living time of the network regardless of the routing protocol. The broken nodes leave a large gap in network topology which PEGASIS is unable to recover. We approach the improvement of network living time by applying the Genetic Algorithm (GA) in reconstructing a more optimized transmission route. A dedicated model of a small-scaled landslide is proposed and simulated together with PEGASIS in MATLAB/Octave. Different scenarios of GA usages are examined in this paper.

The backgrounds of PEGASIS, GA are introduced in Section 2. Methodology, landslide model, and simulation setup are presented in Section 3. Section 4 shows the simulation result and Section 5 will give a conclusion.

\section{Related Works}

\subsection{PEGASIS}

PEGASIS is a chain-based routing protocol for WSN, introduced by S. Lindsey and C.S. Raghavendra [10]. It assumes that all nodes have global knowledge about the location of the whole network. The furthest node from the sink is selected as the first member of the chain. In the non-member set of nodes, the closest node is chosen to be the next member in the chain. The newly added node is then set to find its closest non-member node. The chain is constructed using this greedy algorithm until all the nodes are added into it. The chain can be reconstructed in the same manner when a node died. Another version of PEGASIS allows bypassing the death nodes and directly connect the nodes locate immediately before and after the death node together [13].

After chain construction, all nodes send their data to the leader of the chain, and the leader node transfer aggregated data of all nodes to the sink node. In each transmission round, only one node in the chain is selected as the leader node. Once a node is selected, it cannot be the leader node in the subsequent rounds until all other nodes have taken this role one time. After the last node has taken the role of leader, all the nodes can be chosen again.

During a transmission round, the position in the chain of leader node is important. If the leader is not located at two ends of the chain, data from the first position node and last position node will be transferred to the leader node in opposite directions. As described in [10], a control token is passed to the first position node. This node transfers its data to its only neighbor, the second node in the chain. The second node receives the data and a token; fuses its data with received data and then transfers to the other neighbor. When the leader node receives the token, it also receives data from all the nodes at the first position side. The token is then passed to the last position node and the same process is conducted. 


\subsection{Genetic Algorithm}

The chain construction of PEGASIS can be regarded as an open-loop Travelling Salesman Problem (TSP) and no specific starting point. Finding the best solution for this famous TSP problem by an exact algorithm requires testing all permutations of nodes arrangement, which cost the running time of solving algorithm reaches the level of time complexity at $\mathrm{O}(\mathrm{n} !)$. Heuristic approaches are seen to yield good enough solutions in a much shorter time. The nearest neighbor algorithm, the greedy algorithm which is employed by PEGASIS also belongs to this category. However, according to [14], the nearest neighbor algorithm is noticed that produces not good enough solutions with some arrangements of nodes.

Genetic Algorithm (GA) is an efficient method to find near-optimal chain [15]. GA is based on the theory of evolution, in which the individuals make use of gene exchanges (crossover), mutation, and selection to have a better set of chromosomes in the population. In the TSP problem, cities play the role of genes and the routes are the chromosomes. Similarly, a solution in PEGASIS is a possible chain with arranged nodes. When the order of the nodes is changed, a new solution is found, and a fitness function is calculated to see if this new solution is better than the previous one. As for PEGASIS, the fitness function is the length of the chain, calculated by sum up the distances between neighbor nodes in order.

GA has been used with PEGASIS in [16] for network arrangements in both 2D and 3D. In the application of landslide monitoring, we will only apply GA for the $2 \mathrm{D}$ case but with other scenarios.

\section{Methodology}

This section introduces the models and setup of the simulation. A network model is presented in this section. It describes the location of the nodes in the simulation. The simulation of PEGASIS includes indispensable radio and energy models, which are introduced in sub-section 3.2. The proposed model for small-scale landslide is presented in 3.3. Sub-section 3.4 and 3.5 give more details of GA and the required setting of GA for simulation. Sub-section $3.6 \mathrm{com}$ bines all the above-mentioned models and setups to form the simulation cases and scenarios. Each combination has different settings and is packed into a protocol. To evaluate the protocols, sub-section 3.7 provides metrics to observe as simulation result.

\subsection{Network Model}

A hillside with a size of $100 \mathrm{~m} \times 100 \mathrm{~m}$ is monitored by a network of 100 sensor nodes. The locations of nodes are randomly generated. A sink node is located at $(50,200)$ to ensure it is not affected by landslide in the monitoring area. In Figure 1 , only sensor nodes are shown.

\subsection{Radio and Energy Model}

The first order radio model in [9] [10] [16] is used in this simulation. Sensor 


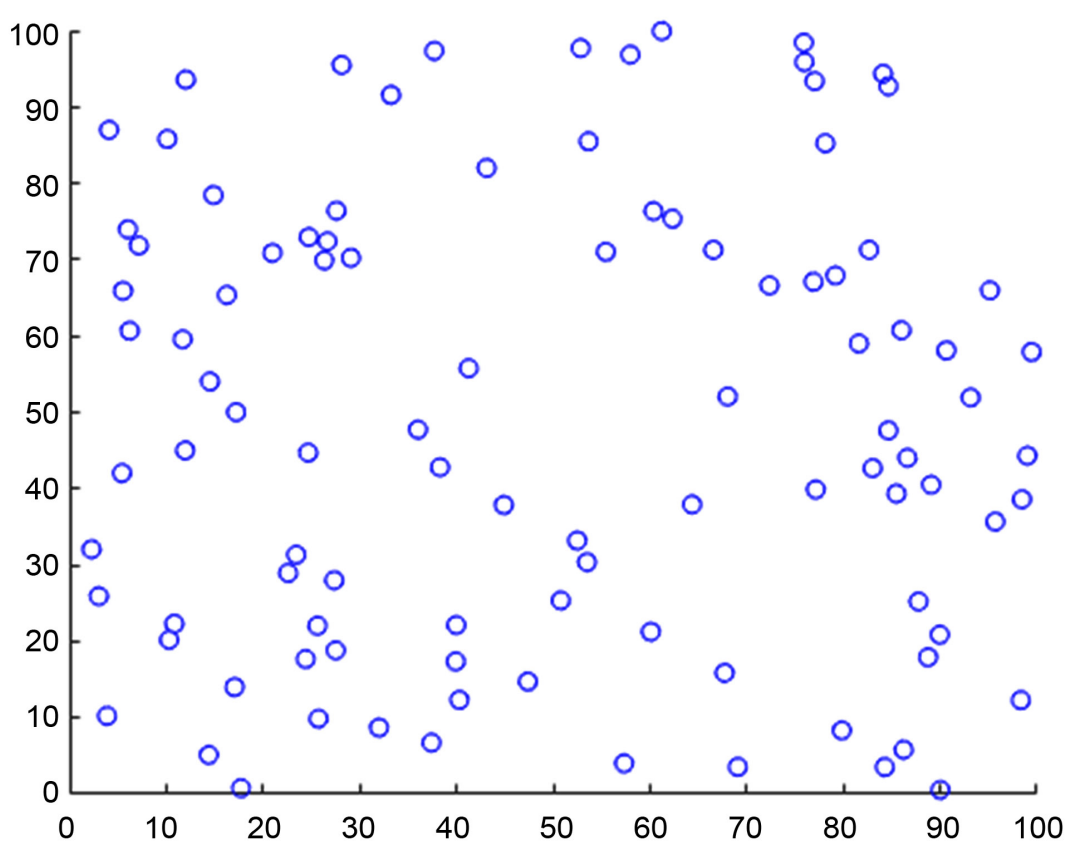

Figure 1. Network model of 100 nodes in a $100 \mathrm{~m} \times 100 \mathrm{~m}$ hillside.

nodes started with an energy amount $E_{0}$ and spend energy through transmitting, receiving, and processing data. Energy is spent mostly in communication, so the energy spent in processing data is counted only when data is aggregated at each forwarding node on the way. Each bit of data in the message costs $E_{\text {elec }}$ to process in both transmitting and receiving. Transmitter also spends $E_{\text {amp }}$ for power amplification. Sending a k-bit message over a distance d requires $E_{T x}$ at transmitter:

$$
E_{T x}=E_{T x-\text { elec }}+E_{\text {amp }}=E_{\text {elec }} * k+E_{\text {amp }} * k * d^{2}
$$

Energy to spend by the receiver:

$$
E_{R x}=E_{R x-\text { elec }}=E_{\text {elec }} * k
$$

Nodes at first and last positions in the chain only spend $E_{T X}$ while other nodes (except the leader node) must spend both $E_{T x} E_{R x}$ and an amount of energy for data aggregation $E_{d x}$. The leader node spends $E_{R x}$ for each side and $E_{T X}$ to transmit data from all nodes to the sink node. Parameters for the energy model are given in Table 1.

The radio channel is assumed to be symmetric, which means data transmitted in both directions between 2 specific nodes cost the same amount of energy under a given signal to noise ratio (SNR).

\subsection{Small-Scale Landslide Model}

The area monitored by the sensor network in Figure 1 is the representation of the slope on a hillside. The foot of the slope is set at the north side and the top is at the south side. The large-scale landslides are suitable with other surveillance methods than sensor networks because they are prominent to be discovered. Furthermore, the whole sensor network can be destroyed by a hundred-meter 
Table 1. Parameters for energy model.

\begin{tabular}{cc}
\hline Parameter & Value \\
\hline$E_{0}$ & $0.5 \mathrm{~J}$ \\
$E_{\text {elec }}$ & $50 \mathrm{~nJ} / \mathrm{bit}$ \\
$E_{\text {amp }}$ & $100 \mathrm{pJ} / \mathrm{bit} / \mathrm{m}^{2}$ \\
$E_{d a}$ & $5 \mathrm{~nJ} / \mathrm{bit}$ \\
$k$ & $2000 \mathrm{bit}$ \\
\hline
\end{tabular}

wide avalanche. In this study, we focus on the landslides of a smaller scale. Some examples are mudslide and debris flow. A small-scale landslide is characterized by parameters:

Type of instance: An instance of the landslide can be a new one, triggered by geomorphic or external factors, which is a primary landslide occurrence. A reactive landslide, delivered by the previous landslide, is called secondary landslide occurrence.

Occurrence probability. A small-scale landslide has probability $p 1$ for the primary occurrence and $p 2$ for secondary occurrence in every round. If a primary landslide starts to occur in round $r$ with probability $p 1$, it may trigger a secondary landslide in round $r+1$ with probability $p 2>p 1$. A secondary landslide at round $r^{2}$ with probability $p 2$ can also trigger another secondary landslide in round $r^{2}+1$ with the same probability. We consider only one concurrent landslide in one round.

Location: We simplify the model of a landslide as a circle with centroid and radius. The coordinate of the centroid of the landslide is randomized, inside the monitoring area.

Area of effect. The area inside the circle with centroid $\left(x_{i}, y_{i}\right)$ and radius $R$ is the area that landslide has an impact. Nodes locate inside the circle have a low possibility of surviving, so we assumed the nodes are destroyed and no longer exchange data with other living nodes.

Direction: The foot of the slope is on the north side and the top is on the south side. Therefore, the landslides will move from the south to the north. The primary landslides start with 0 degrees to the north. The direction of secondary landslide changes randomly after each round, from -30 to +30 degrees of its previous inducing landslide.

Speed: The primary landslides start with no initial velocity. The secondary landslides have velocity ranging from 0 to 10 meters per round. The movement speed can describe the steepness of the slope. The steeper the slope, the higher the velocity.

According to [17], the occurrence probability depends on local geographical features and ranges from $1 \%$ to $81 \%$. Without loss of generality, for each instance of the landslide, the details of location, area of effect, direction, and speed are randomly generated. This depicts various cases that may happen in different conditions of real slopes. The parameters to simulate small-scale landslides are 
shown in Table 2.

Figure 2 shows the network of nodes connected in the PEGASIS chain and the representation of landslides. The nodes are small blue circles and the centroid of each landslide are red rectangles. The random-sized circles around the centroids show the area of effect of landslides. The nodes inside the circles are marked in red.

\subsection{Genetic Algorithm}

To generate a near-optimal solution, GA iterates its process. The process includes selection, crossover, mutation, and fitness computation. The population is generated before the iteration is carried out. The following GA implementation of Kirk [18] does not use crossover but mutation still allows finding new and better solutions.

Initial Population: Each permutation of 100 nodes is a solution of a chain building problem. To create a population of $n$ solutions, we randomly choose $n$ permutations of the nodes. The order of the nodes in each solution is the order of nodes in the chain. Population $n$ is adjusted to be a multiple of 4 to prepare for mutation.

Table 2. Landslide simulation parameters.

\begin{tabular}{cc}
\hline Parameters & Value \\
\hline Primary occurrence probability & $5 \%$ \\
Secondary occurrence probability & $35 \%$ \\
Coordinates of centroid & $(0,0)-(100,100)$ \\
Radius & $0-10 \mathrm{~m}$ \\
Primary occurrence direction & 0 degree \\
\hline
\end{tabular}

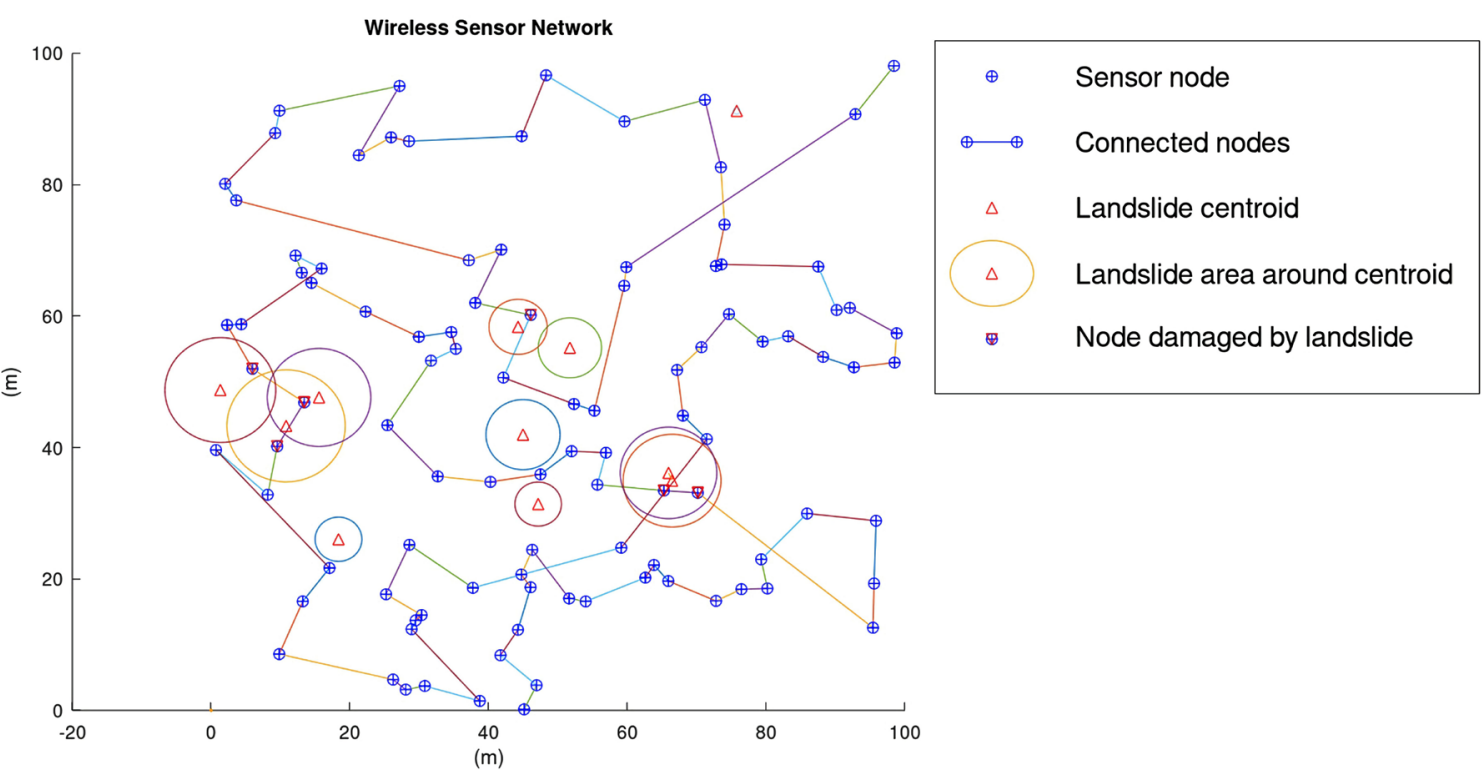

Figure 2. PEGASIS chain and landslide representation. 
Fitness Function: The fitness function in the routing problem is the total length of the route. For each solution in the population, the length of the route is calculated.

Selection: Chooses the best solution in the solution set. Here the route with the shortest length is chosen. The length of this solution is set as the global minimum value.

Mutation: After randomizing the order of solutions in the solution set, every 4 solutions are chosen as a group. The best route of 4 routes in each group is chosen as the main object for mutation. Others will be replaced with the results of Swap, Flip, and Slide operations.

- Swap operation: In the chain, 2 insertion points are randomly selected. The nodes at these points are swapped while other nodes are not changed.

- Flip operation: In the chain, 2 insertion points are randomly selected. The order of all the nodes between these nodes (including nodes at 2 selected points) is reversed.

- Slide operation: In the chain, 2 insertion points are randomly selected. The part between these points is split into two sub-parts. The first includes only the first node, and the second part contains the remaining nodes behind. These two sub-parts change positions, with an effect of the first node slides over the other nodes from the first point to the last point.

After the mutation phase is performed, the population is updated, the fitness function is calculated again. The process from the selection phase is repeated until the maximum iteration is reached. The best route found at the end of the GA process is a near-optimal solution of the chain building. The flowchart of GA is shown in Figure 3.

We apply the GA implementation with modification: In the initial population, the chain generated by PEGASIS nearest neighbor algorithm is used as one solution in the population. This ensures the best solution is at least as good as the result of PEGASIS.

\subsection{Setting for Genetic Algorithm}

Two important parameters of GA are the size of the population and the number of iterations. As the number of iterations increases, better solutions are found. However, as the algorithm approaches the optimal solution, the chance to find a better one does not increase anymore. For a different number of nodes in the chain, the number of generatable solutions varies and it affects the time needed for finding in solution population. In the following examples, population size is set at 100 .

Figure 4 shows the improvement of GA after 5000 iterations with random node locations. After 200 iterations, GA does not find any improvement in the case of 20 nodes. For the case of 40 nodes are around 750 iterations and more iterations are needed for larger sets of nodes. The more nodes in the chain, the longer this process is. 


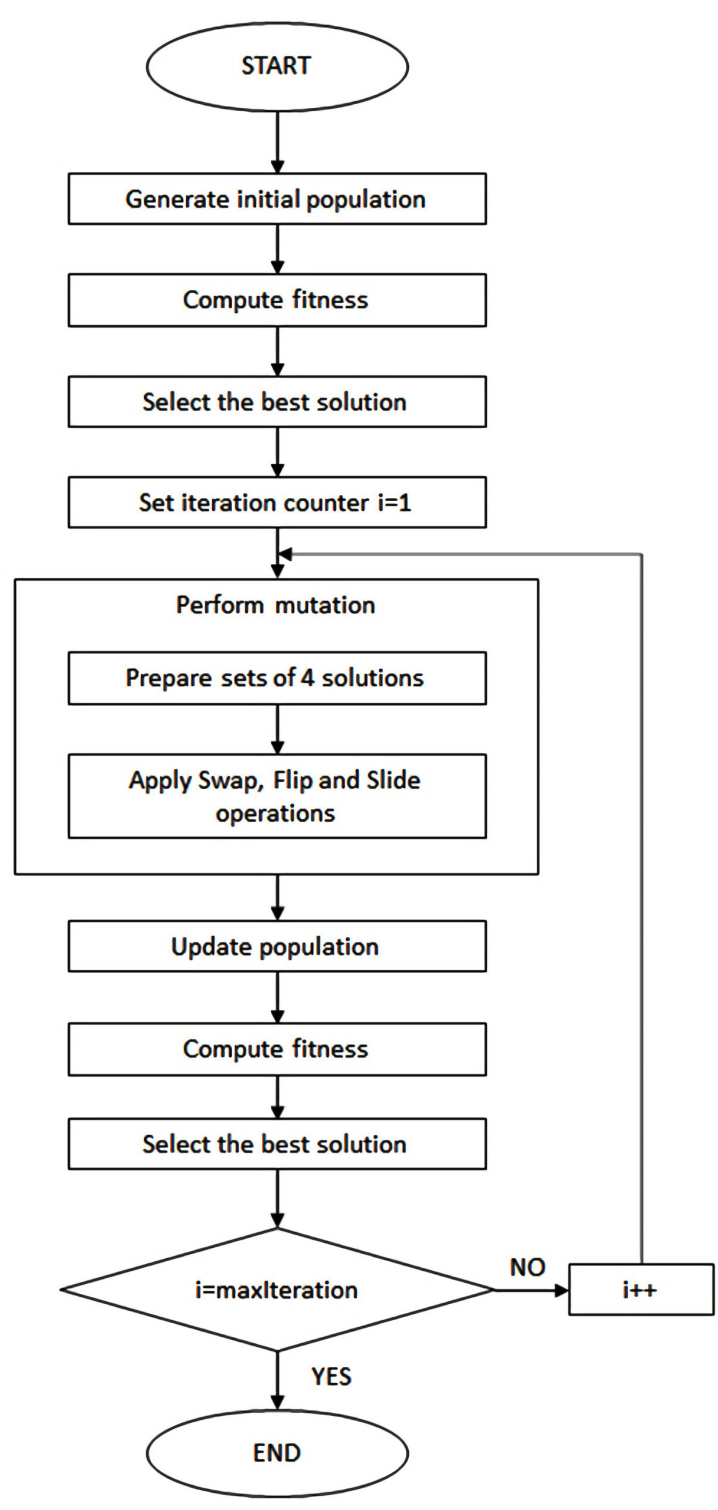

Figure 3. Genetic algorithm flowchart.

Figure 5 shows the improvement of GA after 5000 iterations with preset order of nodes in the chain. As the chain is constructed by the nearest neighbor algorithm, which is used by PEGASIS, the initial length is significantly improved. The effectiveness of GA decreases when the number of iterations increases. Finding a solution, which is better than the current best one, is more difficult. For a set with a low number of nodes, reaching the optimal route is feasible with a small number of iterations. It is more efficient to stop GA sooner than set with a higher number of nodes.

From the test results, we try to set the number of iterations as in (3):

$$
N_{i}=n^{2} / 2
$$

In this equation, $N_{i}$ is the number of iterations and $n$ is the current number of nodes. 


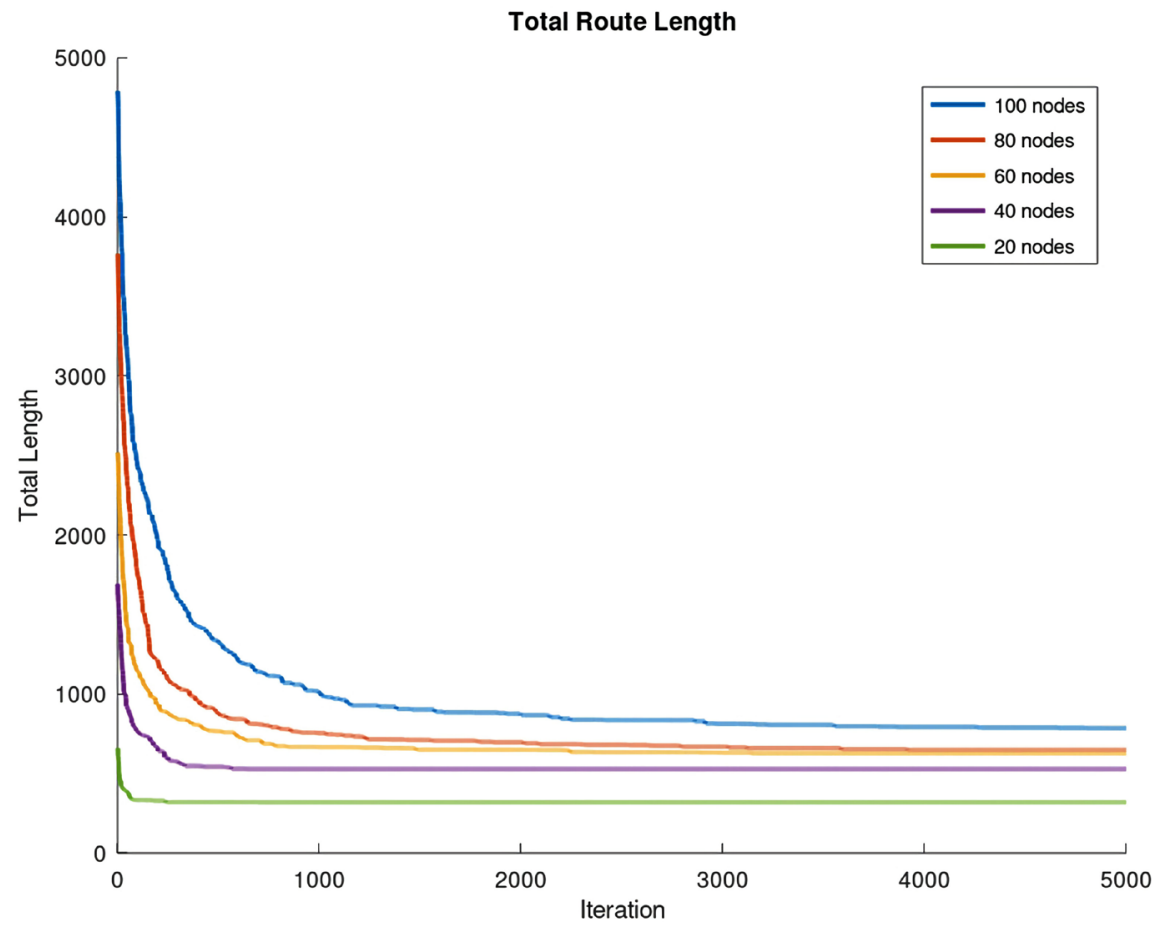

Figure 4. Route length improvement of GA.

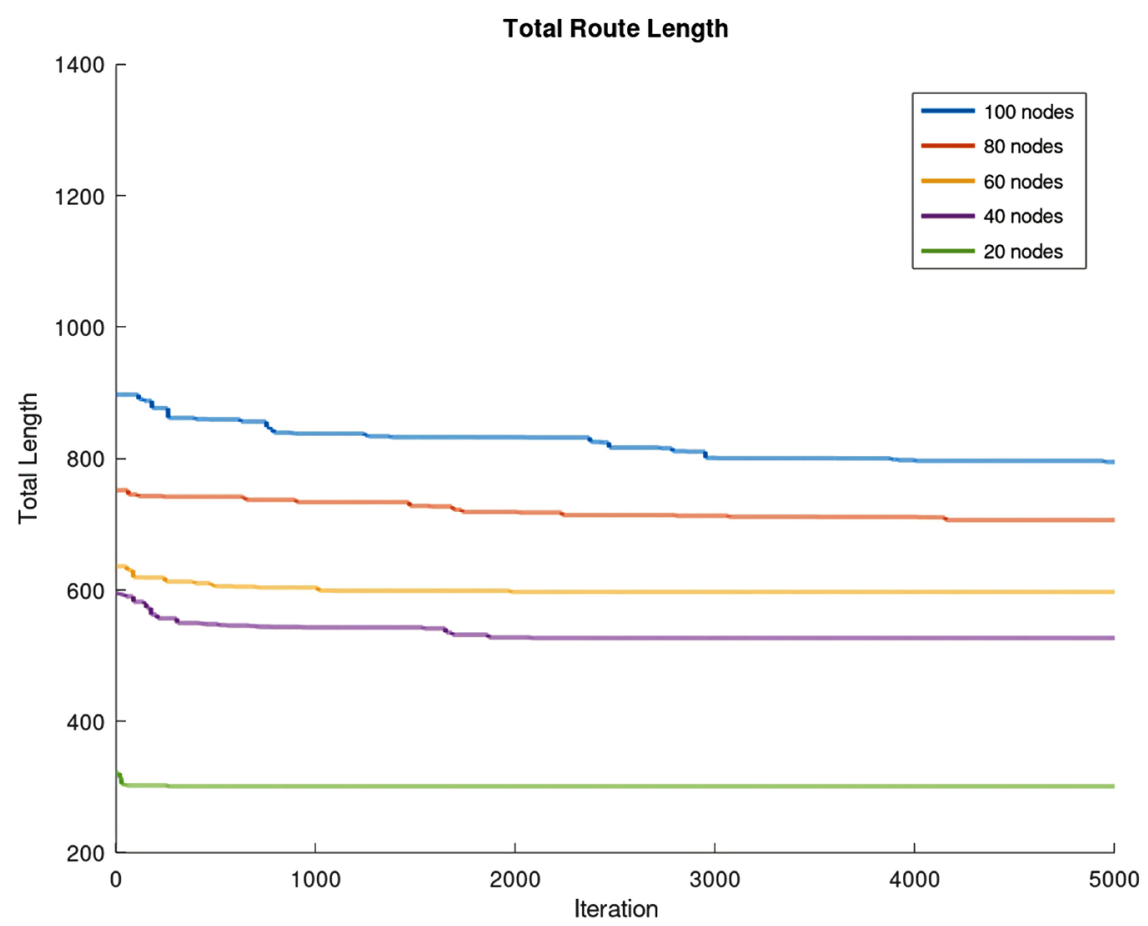

Figure 5. Route length improvement of GA with PEGASIS chain.

\subsection{Simulation Scenarios}

The improvement of GA on PEGASIS has been tested in [16] in one scenario: apply GA to find a better chain than the nearest neighbor, and then running PEGASIS with that starting route. In this paper, other usages of GA are applied 
for 3 cases: Without landslide, with the occurrences of landslides for the whole operation time, and with the occurrence of a landslide when only more than half of the nodes are alive. The setting for each GA usage scenario is shown in Table 3. In the scenario that uses GA after a fixed number of nodes dropped, we test the ability to rebuild the chain after a considerable change in the network. The iteration number follows the Equation (3). In the last protocol, we assume that no landslide happens after $50 \%$ of the total nodes are dead, regardless of the cause. This case is to test the ability to rearrange the network without the interference of landslides.

In typical landslide monitoring systems, the data processing task and data monitoring task must be taken at the same time and the system must be updated with information every few minutes. This allows a shorter time for finding a better route. Depends on the system, the time between 2 rounds may differ but it is limited by the processing time of the computer. Therefore, in scenarios that use GA in every round of PEGASIS, the number of iterations is significantly reduced. The population is also reduced by $50 \%$ to keep the solution set large enough.

\subsection{Metrics}

\subsubsection{Network Lifetime and Node Death Time}

For no landslide cases, network lifetime indicates how long the network can operate by counting the rounds and the number of nodes operating in each round. There are 3 important indexes to consider. For all these indexes, the longer the lifetime, the better the protocol is.

- First node death (FND): the round that records the first instance when the number of nodes drops below the total number of nodes.

- Half node death (HND): the round that records the first time the number of nodes drops to or below $50 \%$ of the total number of nodes.

Table 3. GA scenarios and settings.

\begin{tabular}{|c|c|c|c|c|}
\hline Scenario & Setting & $\begin{array}{c}\text { Protocol } \\
\text { (without Landslide) }\end{array}$ & $\begin{array}{c}\text { Protocol (with } \\
\text { Landslide, all nodes) }\end{array}$ & $\begin{array}{c}\text { Protocol (with } \\
\text { Landslide, half nodes) }\end{array}$ \\
\hline PEGASIS without GA & No setting for GA & PEGASIS & PEGASIS-LS & PEGASIS-LSH \\
\hline $\begin{array}{l}\text { GA is used before the first } \\
\text { round of PEGASIS }\end{array}$ & $\begin{array}{l}\text { Number of iterations: } 5000 \\
\text { Population: } 100\end{array}$ & PEGASIS-GA & PEGASIS-LS-GA & PEGASIS-LSH-GA \\
\hline $\begin{array}{c}\text { GA is used in every round } \\
\text { of PEGASIS }\end{array}$ & $\begin{array}{c}\text { Number of iterations: } 10 \\
\text { Population: } 50\end{array}$ & PEGASIS-GA-E1R & PEGASIS-LS-GA-E1R & PEGASIS-LSH-GA-E1R \\
\hline $\begin{array}{l}\text { GA is used after a fixed } \\
\text { number of nodes dropped }\end{array}$ & $\begin{array}{c}\text { Number of iterations: }\left(n^{2}\right) / 2 \\
\text { Population: } 100 \\
\text { Number of nodes dropped: every } 20 \text { nodes }\end{array}$ & PEGASIS-GA-E20N & PEGASIS-LS-GA-E20N & PEGASIS-LSH-GA-E20N \\
\hline $\begin{array}{l}\text { GA is used both before the } \\
\text { first round and in every } \\
\text { round of PEGASIS }\end{array}$ & $\begin{array}{l}\text { Number of iterations: } \\
\text { - First GA: } 5000 \\
\text { - Every round GA: } 10 \\
\quad \text { Population: } \\
\text { - First GA: } 100 \\
\text { Every round GA: } 50\end{array}$ & PEGASIS-GA-1RER & PEGASIS-LS-GA-1RER & PEGASIS-LSH-GA-1RER \\
\hline
\end{tabular}


- Last node death (LND): the last round that the last node is still in operation. This also indicates the lifetime of the network.

For landslide cases, FND is only considered with nodes that stop working because of energy depletion. LND is considered for all nodes while HND is not observed.

\subsubsection{Energy Consumption}

Energy consumption shows the cumulative energy spent by all the nodes over time. In non-landslide cases, the nodes stop working after their energy elapsed, then when all the nodes are dead, all the energy is spent. In landslide cases, nodes get destroyed during operation do not spend all their energy, so the energy spent in the network after LND is not the total energy that all the nodes have. The less energy spent every round, the longer the network lifetime.

\subsubsection{Route Length}

Route length has a direct impact on energy consumption and indirect impact on network lifetime. A route is represented by a chain of nodes that are arranged in order. The total length of the route is calculated by the sum of the length of all segments from the first node to the last node of the chain:

$$
D=\sum_{i=1}^{n-1} \sqrt{\left(x_{i}-x_{i+1}\right)^{2}+\left(y_{i}-y_{i+1}\right)^{2}}
$$

with $n$ is the total number of nodes in the chain, $i$ is the order of each node in the chain, and $x$ and $y$ are coordinates of the corresponding node.

The improvement of route length by applying GA:

$$
D_{\text {imp }}=D_{1}-D_{2}
$$

Here, $D_{1}$ and $D_{2}$ are the lengths of the route before and after GA is applied, respectively. The length improvement is recorded every round accumulatively.

\section{Results}

The simulation results are shown for each case separately. The case without landslide occurrence is in 4.1. The case with landslide occurrence for the whole simulation is presented in 4.2. Sub-section 4.3 shows the result of the case with landslide occurrence for the first half of the nodes. In sub-section 4.4, some common result features are summarized after all the cases are covered.

\subsection{Case without Landslide Occurrence}

Figure 6 shows the operating nodes and lifetime of the network. Without GA in the first round, FND of PEGASIS and PEGASIS-GA-E20N are the same and appear very soon. Even GA is used, PEGASIS-GA-E20N does not have significant improvement in LND. Before HND, the remaining 3 protocols show better performances but applying GA only boosts LND a little (Table 4).

Figure 7 and Figure 8 show energy consumption over time. During the simulation, most of the scenarios of PEGASIS with GA protocols showed better performance than original PEGASIS, except PEGASIS-GA-E20N. Although 


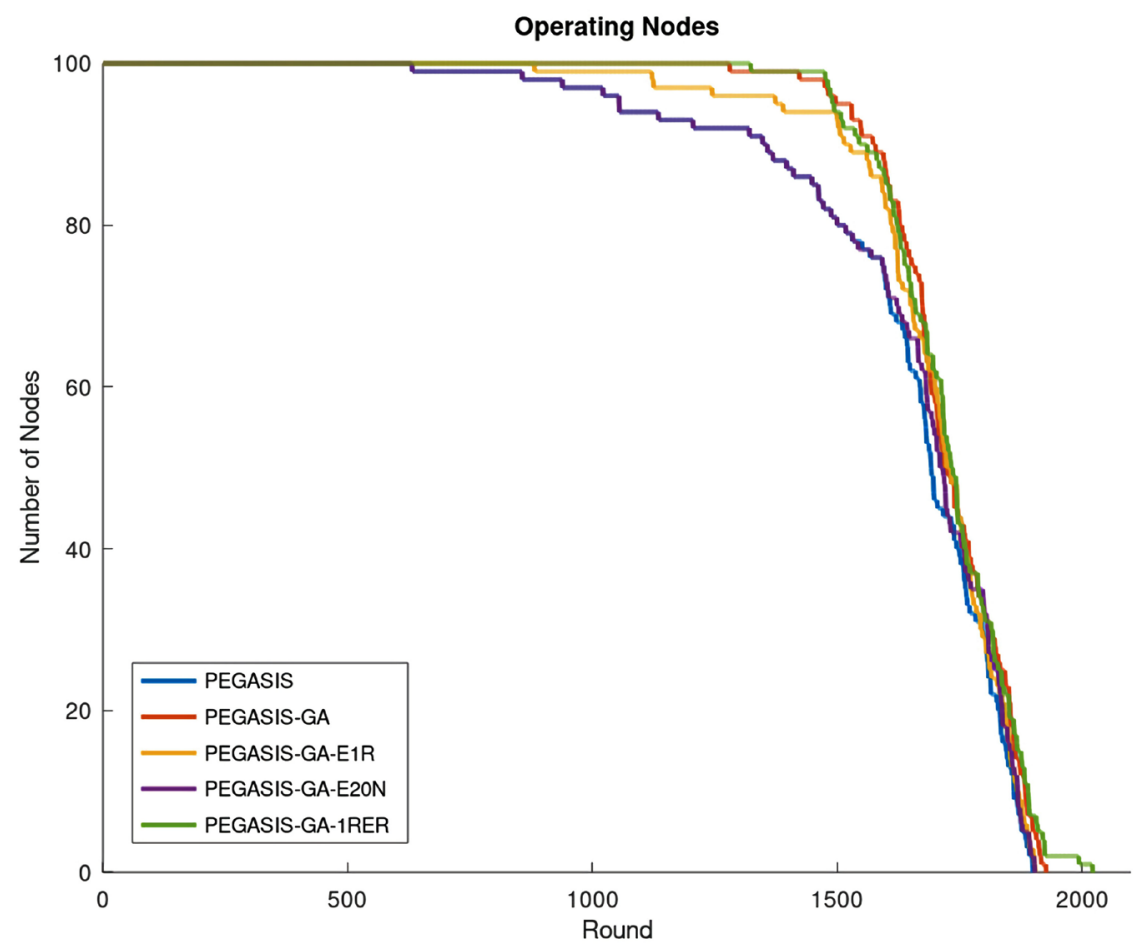

Figure 6. Operating nodes over time, no landslide case.

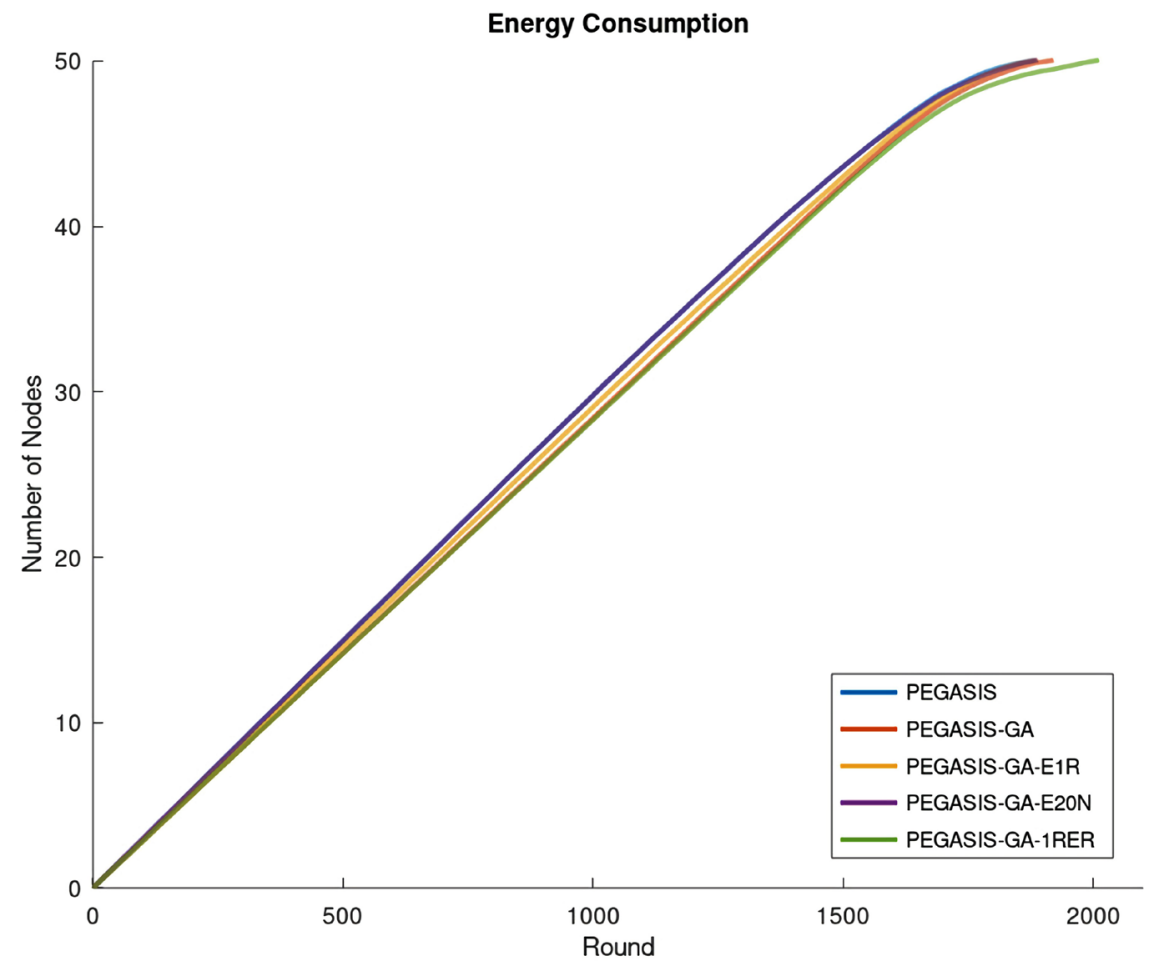

Figure 7. Energy consumption, no landslide case.

PEGASIS-GA-E20N spends slightly less energy than PEGASIS from around round 1600, that is not enough to get past PEGASIS-GA in terms of LND. With a good starting route and continuous finding of better solutions, no surprise that 


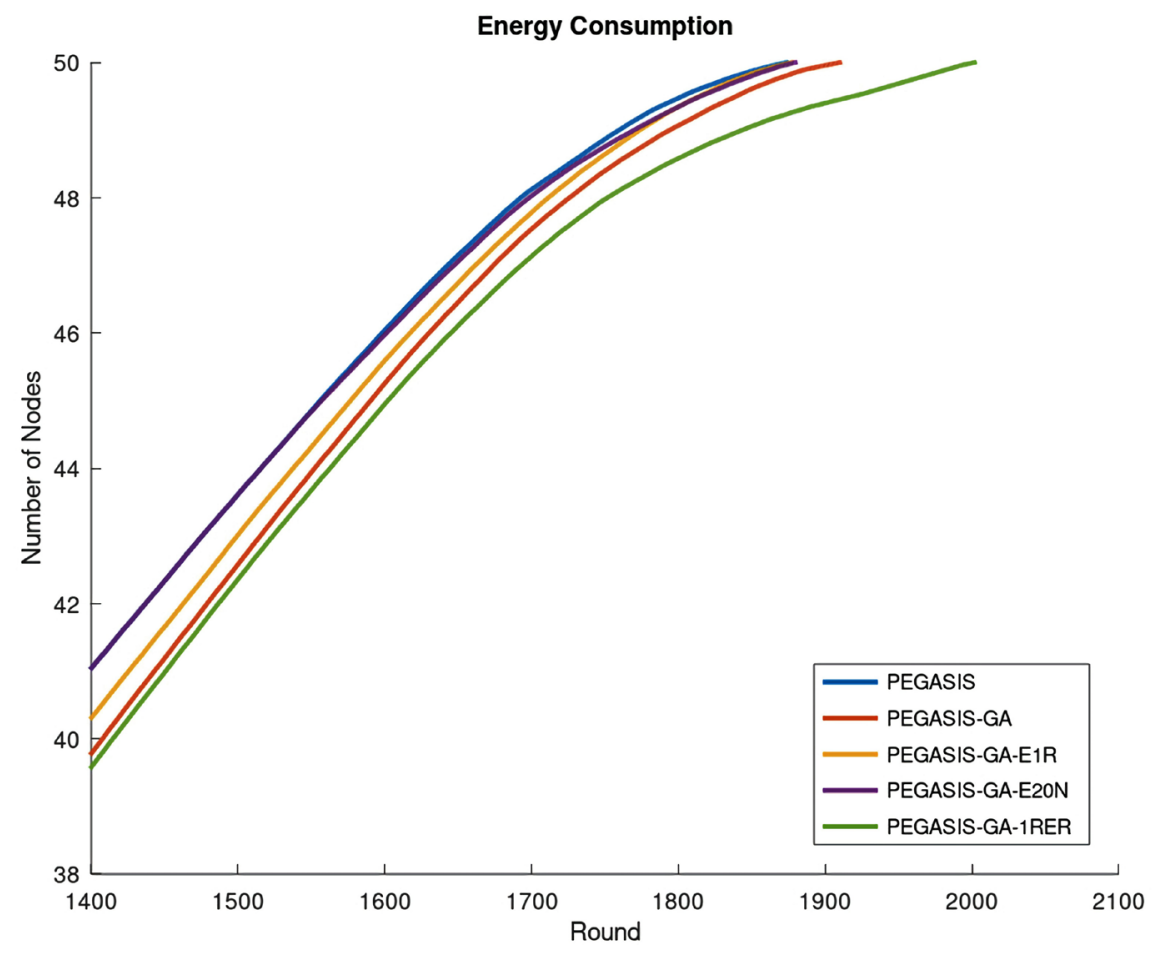

Figure 8. Energy consumption, no landslide case (from round 1400).

Table 4. Lifetime, no landslide cases.

\begin{tabular}{cccc}
\hline Routing Protocol & FND & HND & LND \\
\hline PEGASIS & 632 & 1692 & 1900 \\
PEGASIS-GA & 1281 & 1718 & 1927 \\
PEGASIS-GA-E1R & 882 & 1723 & 1905 \\
PEGASIS-GA-E20N & 632 & 1710 & 1904 \\
PEGASIS-GA-1RER & 1324 & 1733 & 2022 \\
\hline
\end{tabular}

PEGASIS-GA-1RER always has the least energy consumption. In this example, PEGASIS-GA-E1R does not have better energy usage than PEGASIS-GA but no guarantee that which protocol is superior.

Figure 9 shows the total route length changing of all scenarios. The GA applied before the first round of PEGASIS helps PEGASIS-GA and PEGASIS-GA1RER reducing $14.58 \%$ and $12.96 \%$ length of starting route (Table 5). At the round 1500, when the PEGASIS-GA-E20N applies GA for the first time, the reduction of route length is considerable. However, when the node numbers drop to 40,20 , the reduction is not larger than PEGASIS. At these points, the number of nodes that die by energy depletion increases rapidly and the route gets shortened as a result. The routes formed by PEGASIS may not be ideal, but the low number of nodes allows the nearest neighbor algorithm to achieve a near optimal solution without complex computation.

The improvement of GA on route length is shown in Figure 10. These graphs show the length of the route that is saved by GA as in Equation (4). PEGASIS- 


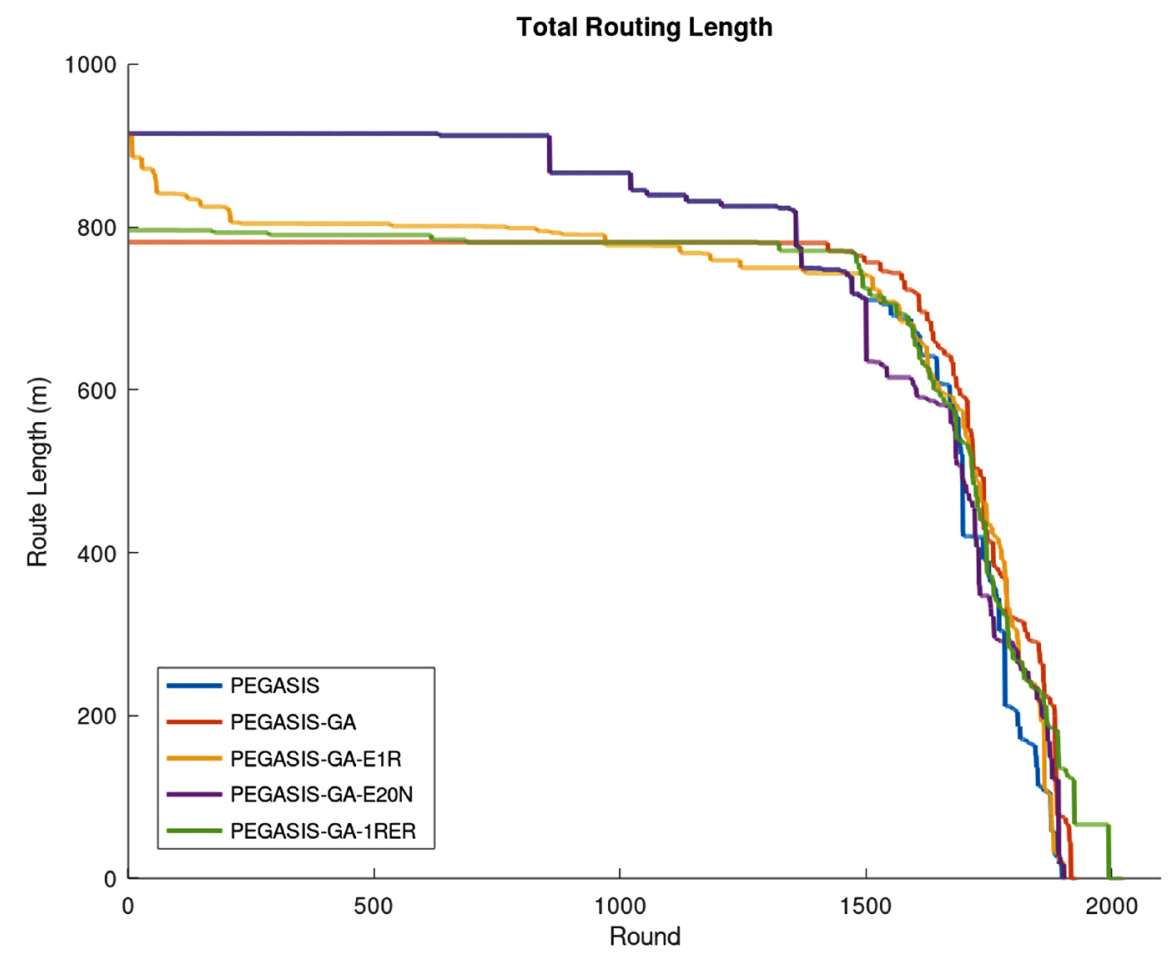

Figure 9. Total route length, no landslide cases.

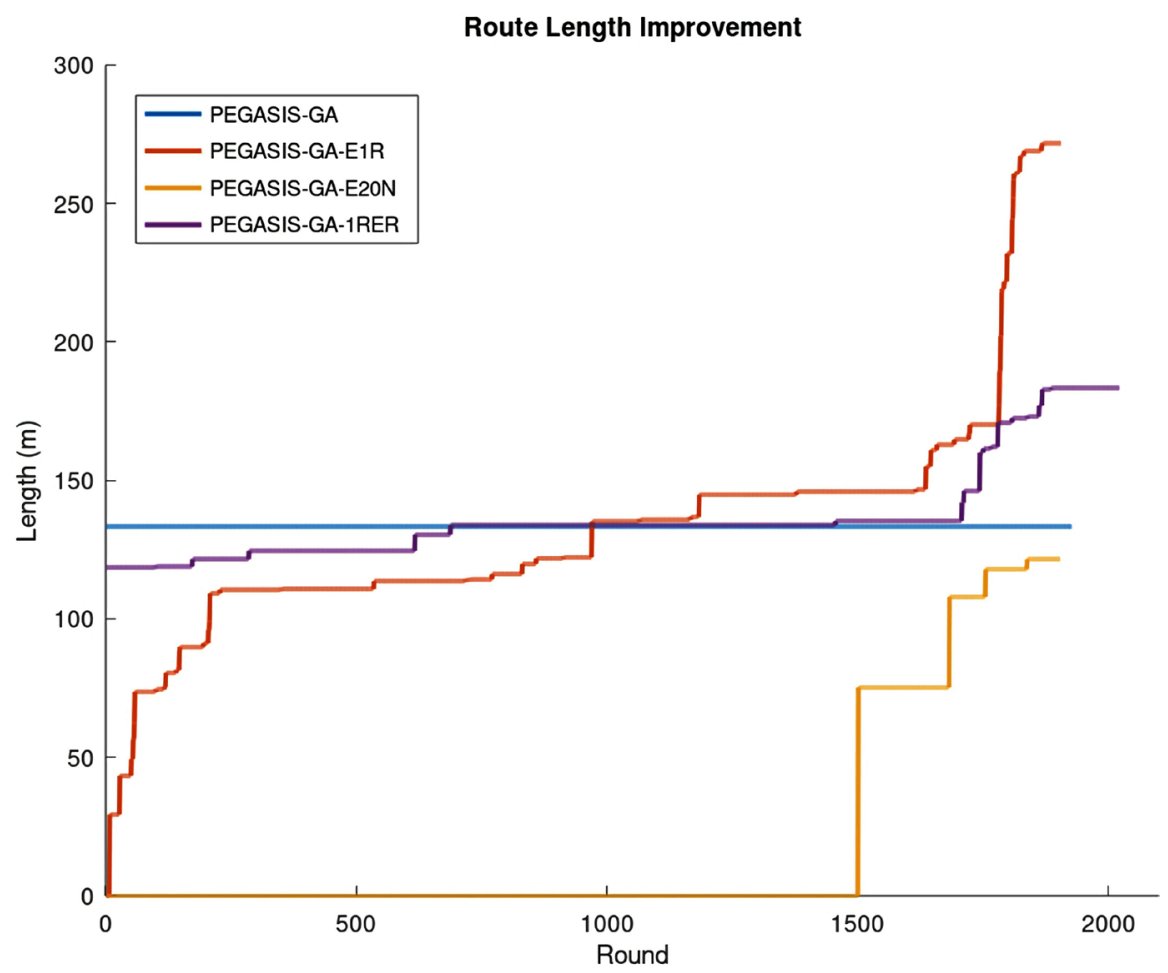

Figure 10. Route Length improvement by GA, no landslide cases.

GA-E1R has a huge improvement at around round 1800. A possible reason is the loss of nodes due to energy depletion makes the topology turns into non-optimal routes. Some non-optimal routes allow GA to improve the route significantly 
Table 5. Landslide simulation parameters.

\begin{tabular}{cc}
\hline Protocol & Total Route Length $(\mathrm{m})$ \\
\hline PEGASIS & 914.86 \\
PEGASIS-GA & 781.46 \\
PEGASIS-GA-E1R & 914.86 \\
PEGASIS-GA-E20N & 914.86 \\
PEGASIS-GA-1RER & 796.25 \\
\hline
\end{tabular}

and all protocols except PEGASIS-GA benefit from this. Finally, PEGASIS-GAE1R improved a total of 271.83 meters of route length. Actual route is not reduced by this length but in comparison, this protocol has potential to reduce an equivalent of $29.71 \%$ of original length.

\subsection{Case with Landslide Occurrence during Operation Time}

In Figure 11, the number of operating nodes is recorded. With the effect of the landslide, the number of nodes decreases from the start of the simulation. As the landslide randomly occurs, different simulations show different results. Without GA, lifetime of PEGASIS-LS reduced $15.32 \%$ compare to PEGASIS. Overall, all the protocols are affected by landslides, and living time is reduced. The number of nodes destroyed by landslides or by out of energy is presented in Table 6 .

Similar to no landslide case, PEGASIS-LS and PEGASIS-LS-GA-E20N have the poorest performance in FND for Energy Depletion nodes. GA applied in the first round still helps PEGASIS-LS-GA and PEGASIS-LS-GA-1RER to have a noticeable good FND compare to other protocols. PEGASIS-LS-GA-E1R does not have as good FND as protocol applied GA at the first round but it is still better than PEGASIS-LS and PEGASIS-LS-GA-E20N. Although PEGASIS-LSGA-E1R has a good start, it does not have a good LND. From the LND and the number of death nodes in both categories, there is a possibility that the less damage receives from landslides, the longer the network lives. With all these uncertainties, the landslides are doubted to have serious effects on all aspects of the routing protocols.

With the heavy impact of landslides, the graphs indicate energy used by PEGASIS-LS-GA-E20N and PEGASIS-LS makes a noticeable turn at the end of the lines (Figure 12). From round 1500, the remaining 3 protocols spend energy at a lower rate, because the number of nodes had decreased to a great extent. PEGASIS-LS-GA survives longer than the rest and has changes to spend energy the most. At the end of simulations, all the protocols spend around 30 to 35 Joules, only $60 \%$ to $70 \%$ of 50 Joules. The unspent amount of energy is wasted in nodes that destroyed by landslides.

In Figure 13, PEGASIS-LS-GA-E20N shows the effects of GA very clearly at 80 nodes remain. However, similar to non-landslide cases, when the number of nodes decreases to 40 or less, the differences between protocols fade off. With a low number of nodes, the chance to form a complex route using the nearest 


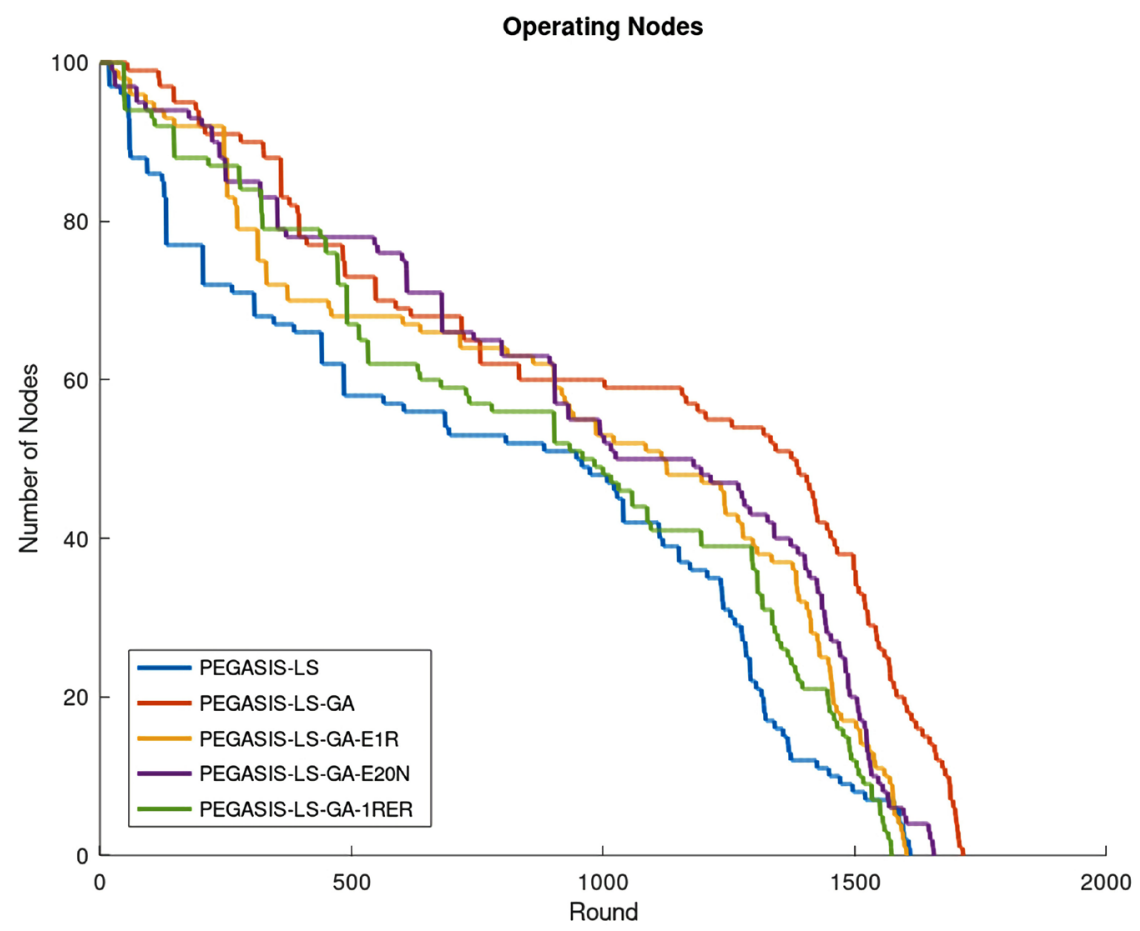

Figure 11. Operating nodes over time, landslide cases.

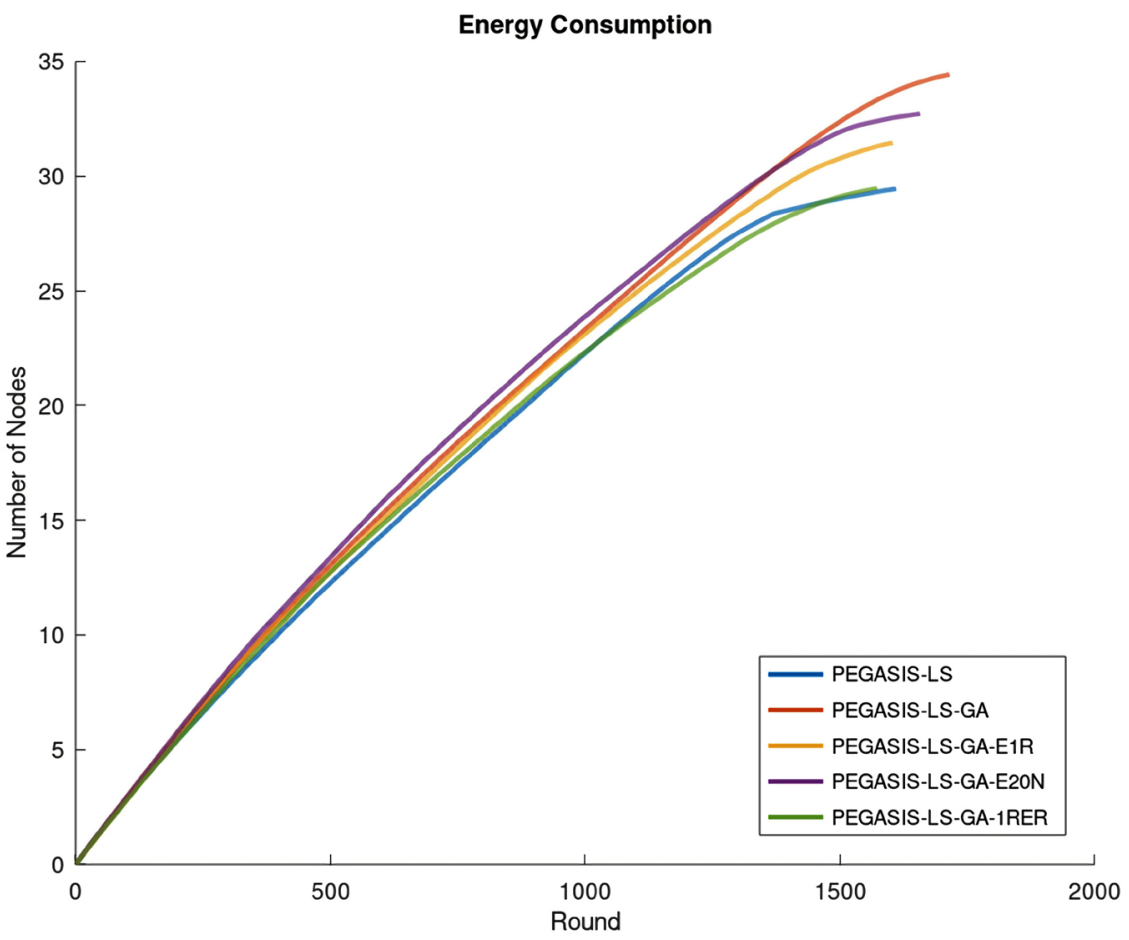

Figure 12. Energy consumption, landslide cases.

neighbor approach is not high. In other words, the route created by PEGASIS is more optimized so the optimal route that generated by GA in PEGASIS-LS-GAE20N is not much better than the original route. The graph of PEGASIS-LS-GAE1R shows that at this point and beyond, a longer route does not mean the less 
Table 6. Nodes death by landslides and energy depletion, landslide case.

\begin{tabular}{ccccccc}
\hline Protocol & $\begin{array}{c}\text { FND } \\
\text { (Landslide) }\end{array}$ & $\begin{array}{c}\text { FND } \\
\text { (Energy } \\
\text { Depletion) }\end{array}$ & $\begin{array}{c}\text { LND } \\
\text { (Landslide) }\end{array}$ & $\begin{array}{c}\text { LND } \\
\text { (Energy } \\
\text { Depletion) }\end{array}$ & $\begin{array}{c}\text { Death by } \\
\text { Landslide }\end{array}$ & $\begin{array}{c}\text { Death by } \\
\text { Energy } \\
\text { Depletion }\end{array}$ \\
\hline PEGASIS-LS & 18 & 603 & 1235 & 1609 & 54 & 46 \\
PEGASIS-LS-GA & 54 & 1255 & 1648 & 1715 & 47 & 53 \\
PEGASIS-LS-GA-E1R & 22 & 1084 & 1450 & 1602 & 59 & 41 \\
PEGASIS-LS-GA-E20N & 23 & 600 & 1452 & 1656 & 54 & 46 \\
PEGASIS-LS-GA-1RER & 47 & 1295 & 1533 & 1572 & 66 & 34 \\
\hline
\end{tabular}

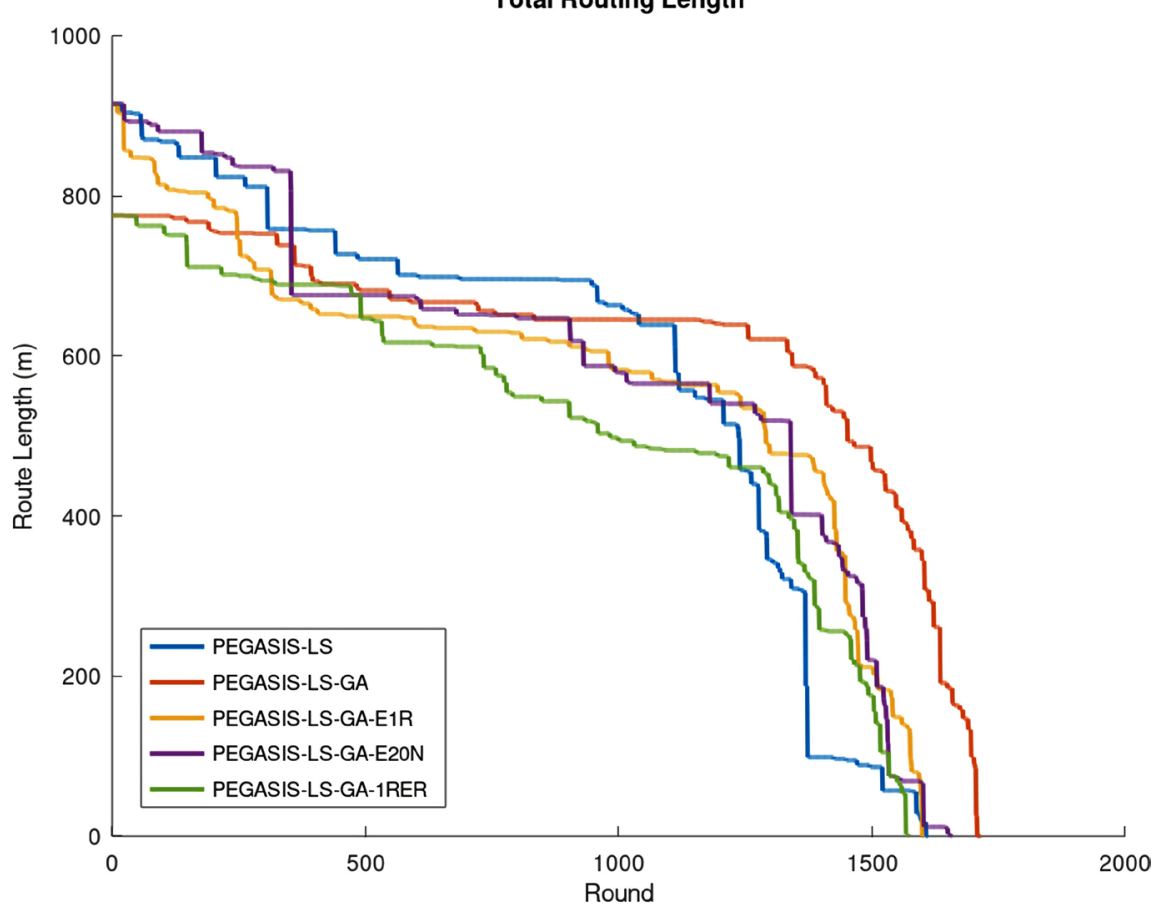

Figure 13. Total route length, landslide cases.

optimal route. It may show that the network luckily survives the damages of landslide and maintain a larger set of nodes. These nodes are the elements that construct a longer route. In Figure 14, although improvements of GA in route length of other protocols are nearly double of PEGASIS-LS-GA, the living time is not prolonged proportionally. PEGASIS-LS-GA-E1R improved equivalent of $31.44 \%$ of route length.

If landslide happens, there is no guarantee that more advanced protocols can perform better than others. The number of nodes that are destroyed by landslides heavily affects the lifetime of the network. Although having the best performance in case of no landslide, PEGASIS-LS-GA-1RER has the poorest result in the case with landslides, retaining only $77.74 \%$ of living time. On the opposite side, PEGASIS-LS-GA-E20N keeps the highest lifetime compare to non-landslide case, at $89 \%$. 


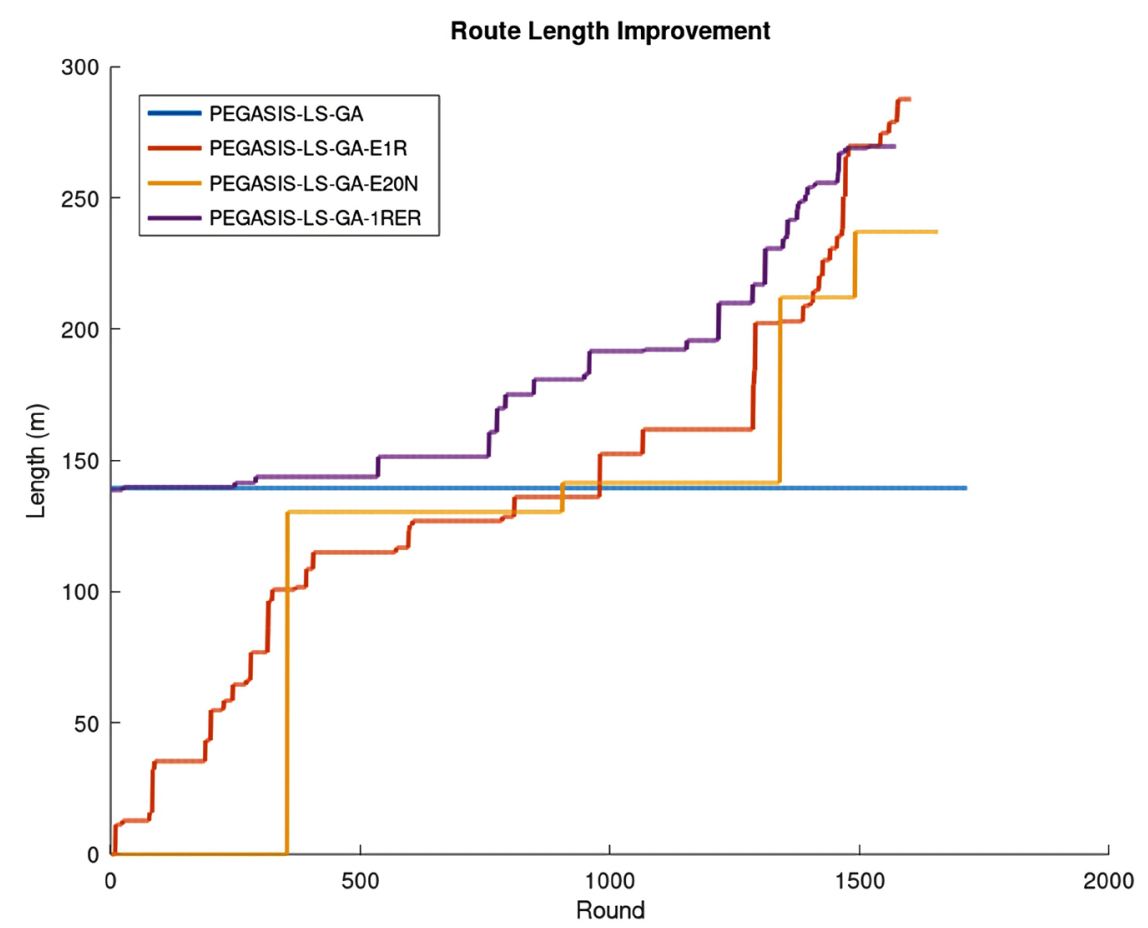

Figure 14. Route length improvement by GA, landslide cases.

\subsection{Case with No Landslide Occurrence after Half of the Nodes Are Dead}

Figure 15 shows the operating nodes over time in the last simulation case. Landslide only happens when the number of nodes is not less than $50 \%$ of the total number of nodes. Therefore, the LND of the landslide is approximately equal to HND of the whole network. The LND (energy depletion) for all protocols in this case concentrate at around 1700 round, similar to the non-landslide case where most of the protocols have LND around 1905. FND (energy depletion) of PEGASIS-LSH-GA-E20N is clearly larger than in previous cases. The node with the lowest energy is possibly damaged by a landslide before reaching its limit.

In Table 7, the number of nodes which are damaged by landslides is inversely proportional to LND (energy depletion). This is also observed in case with landslide occurrence during operation time (Table 6). The protocol with the best LND is PEGASIS-LSH-GA-E1R with living time reach $90.76 \%$ of LND in non-landslide cases.

PEGASIS-LSH-GA and PEGASIS-LSH-GA-1RER show that they are the best in saving energy, thanks to GA applied in the first round (Figure 16). However, the best LND belongs to PEGASIS-LSH-GA-E1R as it is not affected heavily by landslides like others. In these cases, energy used by all the protocols is also at $60 \%-70 \%$ of total energy.

Figure 17 and Figure 18 show the route length over time. The result of route length improvement by GA is similar to the previous landslide case, in which the performance of PEGASIS-LSH-GA only reaches about half of other protocols. 


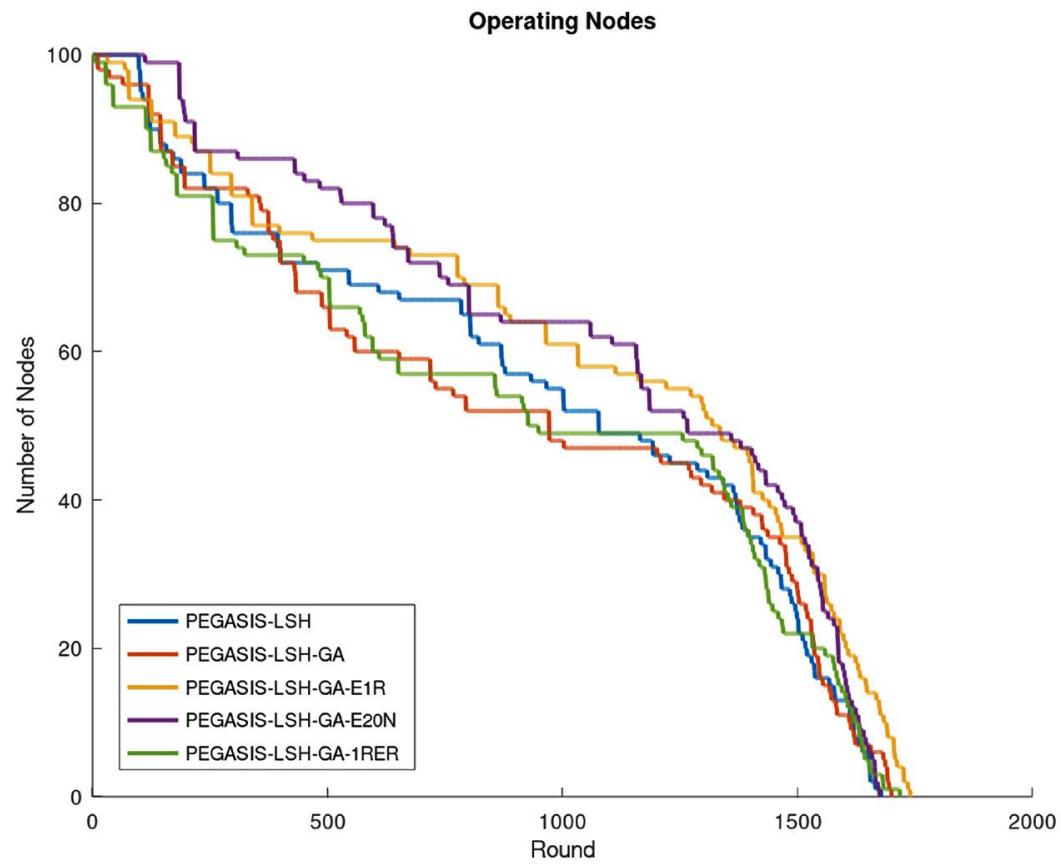

Figure 15. Operating nodes over time, cases with landslide in the first half of nodes.

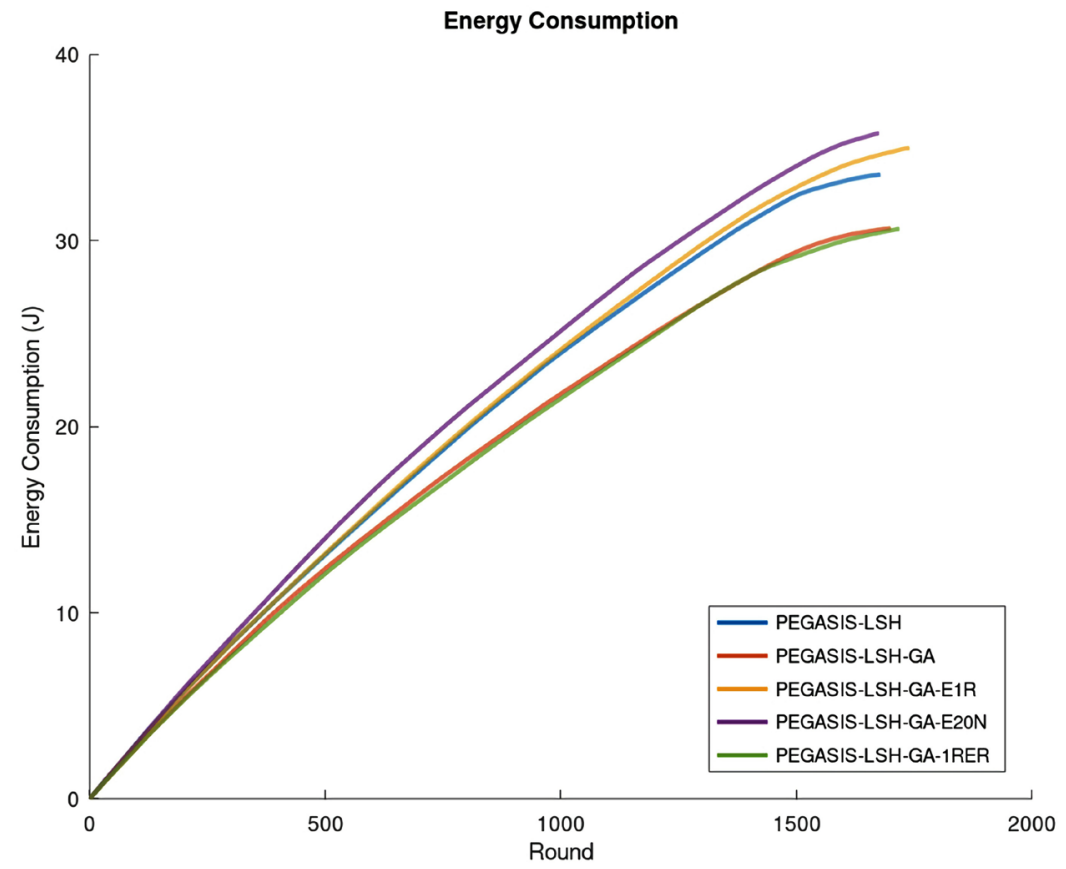

Figure 16. Energy consumption, cases with landslide in the first half of nodes.

Table 7. Nodes death by landslides and energy depletion, landslide case, half nodes.

\begin{tabular}{ccccccc}
\hline Protocol & $\begin{array}{c}\text { FND } \\
\text { (Landslide) }\end{array}$ & $\begin{array}{c}\text { FND } \\
\text { (Energy } \\
\text { Depletion) }\end{array}$ & $\begin{array}{c}\text { LND } \\
\text { (Landslide) }\end{array}$ & $\begin{array}{c}\text { LND } \\
\text { (Energy } \\
\text { Depletion) }\end{array}$ & $\begin{array}{c}\text { Death by } \\
\text { Landslide }\end{array}$ & $\begin{array}{c}\text { Death by } \\
\text { Energy } \\
\text { Depletion }\end{array}$ \\
\hline PEGASIS-LSH & 98 & 608 & 1076 & 1677 & 46 & 54 \\
PEGASIS-LSH-GA & 11 & 1002 & 971 & 1699 & 52 & 48 \\
\hline
\end{tabular}




\section{Continued}

\begin{tabular}{ccccccc}
\hline PEGASIS-LSH-GA-E1R & 31 & 642 & 1318 & 1729 & 45 & 55 \\
PEGASIS-LSH-GA-E20N & 111 & 1358 & 1265 & 1674 & 51 & 49 \\
PEGASIS-LSH-GA-1RER & 3 & 1255 & 948 & 1717 & 51 & 49 \\
\hline
\end{tabular}

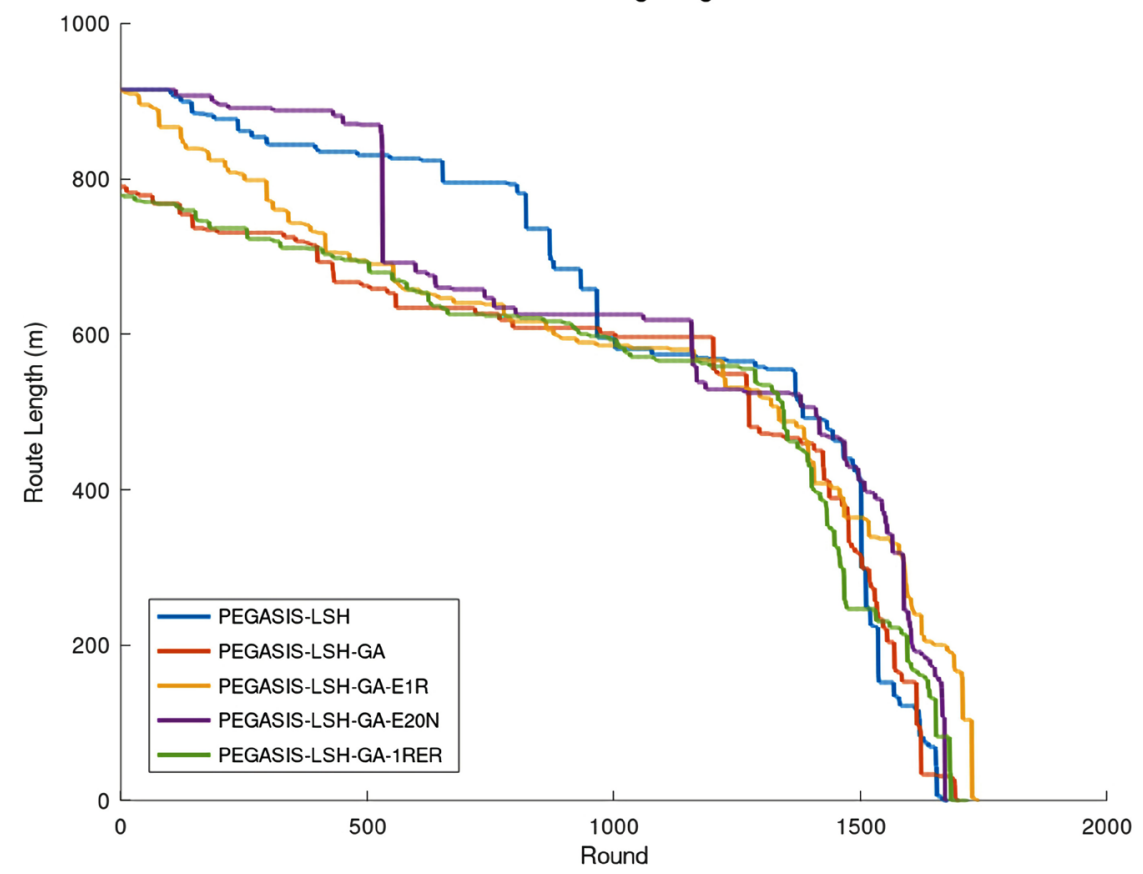

Figure 17. Total route length, cases with landslide in the first half of nodes.

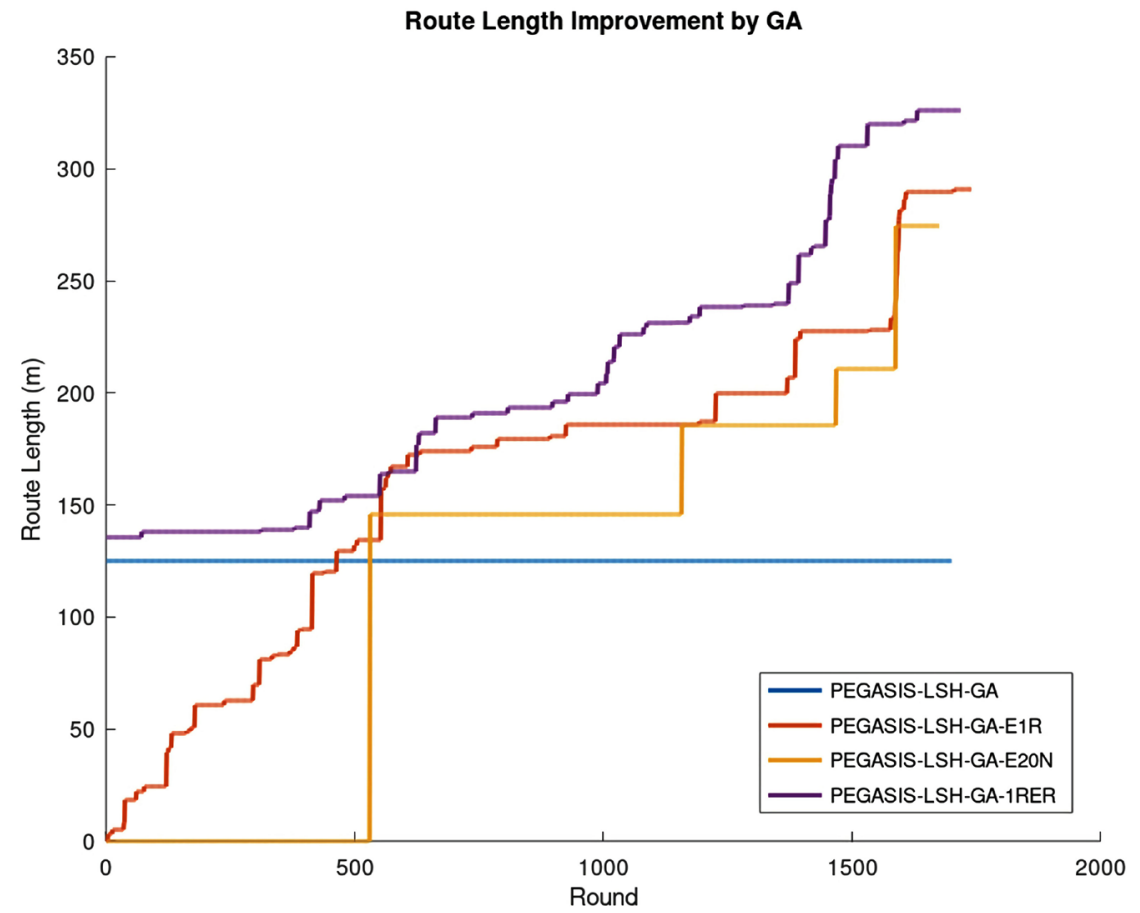

Figure 18. Route length improvement, cases with landslide in the first half of nodes. 
However, the result does not imply that this protocol is inferior to others. While PEGASIS-LSH-GA-E1R and PEGASIS-LSH-GA-E20N have equivalent improvement level at around $31 \%$ of original route length, PEGASIS-LSH-GA-1RER has the higher level at $35.64 \%$.

\subsection{Summary of Simulation Cases}

From the simulation results, it is possible to see the effect of landslides on the network, with or without any method of improving route length and living time. The following points have been observed:

- When landslide happens, nodes are damaged, both network lifetime and route length change accordingly. When landslide does not occur, all protocols are able to maximize both parameters.

- For most of the cases, the number of nodes destroyed by landslides affects the living time of the network. The more nodes are damaged, the shorter the network living time is.

- Having GA applied before the first round is a good start, but it does not maintain a good performance of the protocol at the end.

- The occurrence location and occurrence time of landslides matter the most, as they may change an important part of the topology.

\section{Conclusions}

In this paper, the simulations have been conducted to evaluate the effect of the landslide on the routing capability of a wireless sensor network. To perform the simulation, we proposed a landslide model that is compatible with the well-known routing protocol PEGASIS. This model allowed assessing the effect of the landslide on the communication of WSN. Simulation results showed that the effect on the living time of the network is noteworthy, regardless of the setting of GA. If the nodes are affected heavily by landslides, the robustness of the network is significantly reduced.

Various usages of GA have been implemented to improve the performance of PEGASIS. The results confirmed that GA helps to extend the FND considerably. This paper investigated more deeply on using GA during every round of operation and found that even the iteration and population of GA is set much smaller, applying GA every round does help to boost LND. A combination of GA before the operation of PEGASIS and in every round is the best setting for extending network lifetime.

This paper considered small-scaled landslides with selected ranges of parameters. In the simulation with landslide cases, protocols had energy cut off to $70 \%$. With route length improvement at $30 \%$ of the original length, the living time of the network was kept up to $90 \%$ in comparison with non-landslide cases. The future works can extend the model to adapt to different slope conditions.

\section{Conflicts of Interest}

The authors declare no conflicts of interest regarding the publication of this paper. 


\section{References}

[1] Othman, M.F. and Shazali, K. (2012) Wireless Sensor Network Applications: A Study in Environment Monitoring System. Procedia Engineering, 41, 1204-1210. https://doi.org/10.1016/j.proeng.2012.07.302

[2] Ramesh, M.V., Kumar, S. and Rangan, P.V. (2009) Wireless Sensor Network for Landslide Detection. Proceedings of the 2009 International Conference on Wireless Networks, Las Vegas, 13-16 July 2009, 89-95. https://doi.org/10.1109/SENSORCOMM.2009.67

[3] Rosi, A., Berti, M., Bicocchi, N., Castelli, G., Corsini, A., Mamei, M. and Zambonelli, F. (2011) Landslide Monitoring with Sensor Networks: Experiences and Lessons Learnt from a Real-World Deployment. International Journal of Sensor Networks, 10, 111-122. https://doi.org/10.1504/IJSNET.2011.042195

[4] Sheth, A., Thekkath, C.A., Mehta, P., Tejaswi, K., Parekh, C., Singh, T.N. and Desai, U.B. (2007) Senslide: A Distributed Landslide Prediction System. ACM SIGOPS Operating Systems Review, 41, 75-87. https://doi.org/10.1145/1243418.1243428

[5] Dixon, N., Spriggs, M.P., Meldrum, P., Ogilvy, R., Haslam, E. and Chambers, J. (2010) Development of a Low-Cost Acoustic Emission Early Warning System for Slope Instability. Proceedings of the 11 th IAEG Congress, Auckland, 5-10 September 2010, 1803-1810.

[6] Intrieri, E., Gigli, G., Mugnai, F., Fanti, R. and Casagli, N. (2012) Design and Implementation of a Landslide Early Warning System. Engineering Geology, 147-148, 124-136. https://doi.org/10.1016/j.enggeo.2012.07.017

[7] Selvam, S., Manisha, A., Vidhya, J. and Venkatramanan, S. (2019) Fundamentals of GIS. In: GIS and Geostatistical Techniques for Groundwater Science, Chapter 1, Elsevier, Amsterdam, 3-15. https://doi.org/10.1016/B978-0-12-815413-7.00001-8

[8] Fell, R., Corominas, J., Bonnard, C., Cascini, L., Leroi, E. and Savage, W.Z. (2008) Guidelines for Landslide Susceptibility, Hazard and Risk Zoning for Land Use Planning. Engineering Geology, 102, 85-98.

https://doi.org/10.1016/j.enggeo.2008.03.022

[9] Heinzelman, W.R., Chandrakasan, A. and Balakrishnan, H. (2000) Energy-Efficient Communication Protocol for Wireless Microsensor Networks. Proceedings of the 33 rd Annual Hawaii International Conference on System Sciences, Maui, Vol. 2, 10 p.

[10] Lindsey, S. and Raghavendra, C.S. (2002) PEGASIS: Power-Efficient Gathering in Sensor Information Systems. Proceedings, IEEE Aerospace Conference, Big Sky, 3.

[11] Manap, Z., Ali, B.M., Ng, C.K., Noordin, N.K. and Sali, A. (2013) A Review on Hierarchical Routing Protocols for Wireless Sensor Networks. Wireless Personal Communications, 72, 1077-1104. https://doi.org/10.1007/s11277-013-1056-5

[12] Tejaswi, K., Mehta, P., Bansal, R., Parekh, C., Merchant, S.N. and Desai, U.B. (2006) Routing Protocols for Landslide Prediction Using Wireless Sensor Networks. 2006 Fourth International Conference on Intelligent Sensing and Information Processing, Bangalore, 43-47. https://doi.org/10.1109/ICISIP.2006.4286057

[13] Zattas, A. (2020) PEGASIS (Power-Efficient Gathering in Sensor Information Systems).

https://www.mathworks.com/matlabcentral/fileexchange/67504-pegasis-power-effic ient-gathering-in-sensor-information-systems

[14] Gutin, G., Yeo, A. and Zverovich, A. (2002) Traveling Salesman Should Not Be Greedy: Domination Analysis of Greedy-Type Heuristics for the TSP. Discrete Applied Mathematics, 117, 81-86. https://doi.org/10.1016/S0166-218X(01)00195-0 
[15] Reeves, C. (2003) Genetic Algorithms. In: Glover, F. and Kochenberger, G.A., Eds., Handbook of Metaheuristics, International Series in Operations Research \& Management Science, Vol. 57, Springer, Boston, 55-82.

https://doi.org/10.1007/0-306-48056-5_3

[16] Somauroo, A. and Bassoo, V. (2019) Energy-Efficient Genetic Algorithm Variants of PEGASIS for 3D Wireless Sensor Networks. Applied Computing and Informatics. https://doi.org/10.1016/j.aci.2019.07.002

[17] Petrea, D., Stefan, B., Rosca, S., Vescan, I. and Fodorean, I. (2014) The Determination of the Landslide Occurrence Probability by GIS Spatial Analysis of the Land Morphometric Characteristics (Case Study: The Transylvanian Plateau). Carpathian Journal of Earth and Environmental Sciences, 9, 91-102.

[18] Kirk, J. (2020) Open Traveling Salesman Problem-Genetic Algorithm. https://www.mathworks.com/matlabcentral/fileexchange/21196-open-traveling-sale sman-problem-genetic-algorithm 